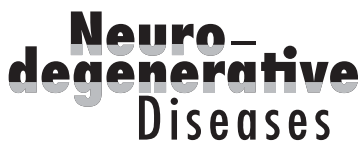

\title{
Tau Immunotherapy and Imaging
}

\author{
Einar M. Sigurdsson ${ }^{\mathrm{a}, \mathrm{b}}$ \\ Departments of ${ }^{a}$ Neuroscience and Physiology, and ${ }^{b}$ Psychiatry, New York University School of Medicine, \\ New York, N.Y., USA
}

\section{Key Words}

Alzheimer's disease · Tauopathies - Tau · Neurofibrillary tangles · Immunotherapy · Immunization - Vaccine

\begin{abstract}
Disappointing findings from recent phase III trials on amyloid- $\beta(A \beta)$ immunotherapy for Alzheimer's disease (AD) have shifted the focus of such treatments to the tau protein. As tau pathology correlates better with the degree of dementia than $A \beta$ plaque burden, it is a more attractive target once cognitive impairments are evident, while $A \beta$ therapies may be better suited for the presymptomatic phase of the disease. Over 12 years ago, we initiated a tau immunotherapy program, seeking to alleviate the functional impairments associated with tau lesions in tauopathies. We have reported that various active and passive tau immunizations diminish tau pathology and improve function, including cognition, in different mouse models. Both extra- and intracellular pathways are likely involved. The antibodies may block the spread of tau pathology via microglial phagocytosis of the antibody-tau complex and facilitate lysosomal tau clearance in neurons after endosomal uptake. We have observed such antibody internalization following intracarotid injection in mice and in various culture models. These include brain slices and primary neurons from tangle mice as well as human neuroblastoma cell lines. Antibody targeting of different intracellular protein aggregates, including $a$-synuclein, $A \beta$ and superoxide dismutase has been reported by others. Now,
\end{abstract}

several laboratories have confirmed and extended our findings using various active and passive tau immunizations in different models, thereby clearly establishing the feasibility of this approach for clinical trials. We are also working on imaging approaches to monitor tau pathology, its consequences and the efficacy of treatments. Dire need exists for such diagnostic methods for tauopathies. Overall, therapies and diagnostic tools targeting tau pathology have a great potential for AD and other tauopathies.

(c) 2013 S. Karger AG, Base

\section{Tau Immunotherapy}

Several clinical trials are assessing the therapeutic benefit of targeting amyloid- $\beta(A \beta)$ in Alzheimer's disease (AD). Many of these are immunotherapies. Findings from the earliest trial suggest that plaque clearance did not halt or slow the progression of dementia, emphasizing the need for alternative targets, further supported by the modest or lack of efficacy observed in recent phase III $A \beta$ antibody trials [1]. Obviously, tau pathology is another important target in $\mathrm{AD}$ and the primary target in other tauopathies. Clearing $A \beta$ may not be sufficient to halt the progression of $\mathrm{AD}$, and pathological tau correlates much better with the degree of dementia than $A \beta$ deposition [2]. Hence, targeting tau may be more effective than removing $A \beta$ once cognitive impairments are evident.

\section{KARGER}

E-Mail karger@karger.com

www.karger.com/ndd
(C) 2013 S. Karger AG, Basel

$1660-2854 / 13 / 0133-0103 \$ 38.00 / 0$
Einar M. Sigurdsson, $\mathrm{PhD}$

New York University School of Medicine, Department of Neuroscience and Physiology Medical Science Building, MSB459

550 First Avenue, New York, NY 10016 (USA)

E-Mail einar.sigurdsson@ nyumc.org 
Our pioneering findings indicate that active immunization with an AD-related phosphorylated tau epitope, Tau379-408[P-Ser396, 404] in JNPL3 P301L tangle model mice reduces brain levels of aggregated tau and slows the progression of the tangle-related behavioral phenotype [3]. We subsequently showed that this vaccine reduces tau aggregates and prevents cognitive decline in three different tests in another tangle model, htau/PS1, that we developed by crossing available models [4]. Furthermore, we demonstrated that passive tau immunotherapy targeting the same epitope is effective as well [5, 6]. Our findings [3, 7-9], and numerous reports of neuronal uptake of antibodies, suggest that intracellular tau aggregates are being cleared [2]. Specifically, we have shown that these antibodies enter the brain, are taken up into neurons primarily via low-affinity $\mathrm{Fc}$ receptors and bind to pathological tau within the endosomal/lysosomal system of neurons [3, 7-9]. In addition, antibody-mediated clearance of extracellular tau/tangles may reduce associated damage and prevent the spread of tau pathology $[10,11]$. Others have reported that different intracellular aggregates, $\alpha$-synuclein, $A \beta$ and superoxide dismutase can be targeted with immunotherapy [reviewed in 2,12 ]. These studies support our findings and interpretations. Most recently, the promise of tau immunotherapy has been confirmed and extended by other groups [13-18], with numerous additional meeting abstracts presented in recent years.

As the tau protein is about ten times the size of the $A \beta$ peptide, is has multiple additional target sites. Which one of these is best to target may depend on the stage of the disease, as it is well known that the tau epitope profile changes over the course of the disease. How prominent the epitope is in the disorder and how specific it is to the pathological state need also to be taken into account when choosing a target epitope. For active vaccines, the immunogenicity of the epitope is also a major consideration as the elderly have an attenuated immune response, and strong adjuvants may lead to severe side effects. Of tau epitopes, phosphoepitopes are the best characterized, and therefore, the obvious first choice for proof-of-concept studies. For active vaccines, a balance between epitope prominence, pathological specificity and immunogenicity is logical in first-generation vaccines. This consideration guided my design and selection, for the initial animal studies, of the prototype vaccine, Tau379-408[PSer396, 404], administered with clinically approved alum adjuvant. Importantly, this adjuvant promotes antibody response over cytotoxic T-cell response, and therefore, is less likely to lead to adverse reactions. It is now important to clarify how narrow this epitope can be while maintaining efficacy, as larger epitopes are more likely to elicit Tcell-related adverse reactions. Mapping the human tau Tcell epitopes remains to be done experimentally, but various computer algorithms can identify likely regions, which depend on the haplotype of the individual. It is also of considerable interest to determine if differences in efficacy/toxicity exist between single-epitope vaccines versus multi-epitope vaccines. If antibody-facilitated clearance of intracellular tau within the endosome-autophagosome-lysosome system turns out to be the major clearance pathway, pan-tau epitopes are also attractive, as the antibodies would then presumably not have access to normal soluble and functional tau within the cytosol. However, if the endocytosed antibodies are released into the cytosol and/or if normal tau turns out to have an important extracellular biological function, targeting such a promiscuous epitope would not be appropriate. While the active approach has certain advantages, it may have autoimmune side effects that can be avoided with narrower/single-epitope vaccines or with passive immunization, which also allows more specific targeting of diseaserelated epitopes. Interestingly, Rosenmann et al. [19] designed a study with the objective to assess if whole recombinant tau protein (no phosphoepitopes) could induce a neuroautoimmune disorder in mice based on their preliminary observation of more tau plasma antibodies in $A D$ versus age-matched controls. Indeed, it led to delayed neurological deficits when administered with two strong adjuvants that are known to promote cytotoxic T-cell responses. For human trials, milder adjuvants that promote antibody response can be complemented with T-helper epitopes attached to a narrow tau epitope to seek an ideal efficacy/safety profile. Within the passive approach, antibody engineering may lead to further improvements.

\section{Single-Chain Variable Fragments for Therapy and Diagnosis}

Specifically, to potentially improve therapeutic efficacy and to develop novel diagnostic markers, with phage display technology, we have generated numerous singlechain variable fragments (scFvs) of monoclonal tau antibodies that we have developed. These smaller entities have certain advantages and disadvantages over tau antibodies and should ideally be developed concurrently. A certain advantage of using scFvs is that because of their smaller size, they may have better access than antibodies to tau aggregates and may therefore be more efficacious 
as therapy or diagnostic markers for tau pathology. The caveat is that this may not be the case if Fc-mediated uptake of tau antibodies into neurons and/or microglia is important for their efficacy. Their main disadvantage as therapy is their presumed short half-life, but well established procedures are available to prolong it and render these molecules more attractive as potential therapy.

On the diagnostic front, antibody detection of abnormal tau in cerebrospinal fluid has shown some promise, suggesting that further development in this arena is warranted. In particular, smaller antibody fragments that bind to tau are attractive as ligands for in vivo imaging to detect tau lesions in patients with $\mathrm{AD}$ or other tauopathies. For such in vivo studies, relatively rapid clearance is considered to be ideal rendering the antibody fragments more attractive than unmodified antibodies. Several scFvs are already being developed as imaging probes against various other targets. In vivo imaging of $A \beta$ plaques using compounds that bind well to $\beta$-sheets is already in clinical use but only a few tau-binding ligands have been identified in preclinical studies [20-23] and some of those have failed in clinical trials [24], while others are still being evaluated [23]. Antibody-based probes, such as those proposed here, are likely to provide greater specificity for detecting tau lesions.

\section{Manganese-Enhanced Magnetic Resonance Imaging}

Several years ago, the Koretsky laboratory introduced a novel MRI approach to directly detect neuronal activity. Manganese-enhanced MRI (MEMRI) utilizes divalent manganese, $\mathrm{Mn}^{2+}$, which enters activated neurons through calcium channels and produces a strong signal enhancement on T1-weighted MRI $[25,26]$. Manganeseenhanced MRI is also emerging as an effective in vivo approach for tracing axonal tracts [26], showing T1-weighted MRI enhancement in the adult and developing mouse brain after subcutaneous, intraperitoneal or intranasal injection of $\mathrm{MnCl}_{2}$ [27-30]. Initial concerns about neurotoxic effects of manganese have been alleviated, since doses required for enhancement in a number of brain regions appear to be safe for mice from the neonatal stage to adulthood with no apparent abnormal behavior, at least over several months $[28,29]$. We have recently reported that this approach allows monitoring of the deterioration of neuronal transport in mice as tau pathology advances with age [29], as well as in vivo detection of the beneficial effects of tau immunotherapy on neuronal transport in the same tauopathy mouse model [30].

Overall, these approaches presented at the 11th International Conference on Alzheimer's and Parkinson's Disease, and briefly discussed here, may lead to novel therapies and diagnostic markers for $\mathrm{AD}$ and related tauopathies.

\section{Acknowledgements}

This work was funded with support from NIH grant numbers NS077239, AG032611 and AG020197. NYU technology on tau immunotherapy and related diagnostics is licensed to and is being codeveloped with $\mathrm{H}$. Lundbeck A/S.

\section{References}

$\checkmark 1$ Aisen PS, Vellas B: Editorial: passive immunotherapy for Alzheimer's disease: what have we learned, and where are we headed? J Nutr Health Aging 2013;17:49-50.

$>_{2} \mathrm{Gu}$ J, Sigurdsson EM: Immunotherapy for tauopathies. J Mol Neurosci 2011;45:690695.

- 3 Asuni AA, Boutajangout A, Quartermain D, Sigurdsson EM: Immunotherapy targeting pathological tau conformers in a tangle mouse model reduces brain pathology with associated functional improvements. J Neurosci 2007;27:9115-9129.

- 4 Boutajangout A, Quartermain D, Sigurdsson EM: Immunotherapy targeting pathological tau prevents cognitive decline in a new tangle mouse model. J Neurosci 2010;30:1655916566.
5 Boutajangout A, Ingadottir J, Davies P, Sigurdsson EM: Passive immunization targeting pathological phospho-tau protein in a mouse model reduces functional decline and clears tau aggregates from the brain. J Neurochem 2011;118:658-667.

-6 Boutajangout A, Rajamohamed Sait HB, Gonzalez V, Sigurdsson EM: Targeting hyperphosphorylated tau protein with a monoclonal antibody at an advanced stage of tau pathology improves cognition in a mouse model. Alzheimers Dement 2011;7(suppl 4):S480-S481.

7 Krishnamurthy PK, Deng Y, Sigurdsson EM: Mechanistic studies of antibody-mediated clearance of tau aggregates using an ex vivo brain slice model. Front Psychiatry 2011;2:59.
$8 \mathrm{Gu}$ J, Sigurdsson EM: Efficacy and mechanisms of antibody-mediated clearance of tau aggregates in brain slice model. Soc Neurosci Meeting Abstract 852.12, New Orleans, 2012.

9 Congdon E, Sait H, Boutajangout A, Sigurdsson EM: Tau antibody uptake into neurons strongly correlates with degree of tau pathology and is primarily receptor-mediated via low affinity Fcgamma receptors. Soc Neurosci Meeting Abstract 852.10, New Orleans, 2012.

10 Clavaguera F, Lavenir I, Falcon B, Frank S, Goedert M, Tolnay M: 'Prion-like' templated misfolding in tauopathies. Brain Pathol 2013; 23:342-349.

11 Walker LC, Diamond MI, Duff KE, Hyman BT: Mechanisms of protein seeding in neurodegenerative diseases. JAMA Neurol 2013;70: 304-310. 
12 Valera E, Masliah E: Immunotherapy for neurodegenerative diseases: focus on alpha-synucleinopathies. Pharmacol Ther 2013;138: 311-322.

13 Boimel M, Grigoriadis N, Lourbopoulos A, Haber E, Abramsky O, Rosenmann H: Efficacy and safety of immunization with phosphorylated tau against neurofibrillary tangles in mice. Exp Neurol 2010;224:472-485.

14 Chai X, Wu S, Murray TK, Kinley R, Cella CV, Sims H, Buckner N, Hanmer J, Davies P, O’Neill MJ, Hutton ML, Citron M: Passive immunization with anti-Tau antibodies in two transgenic models: reduction of Tau pathology and delay of disease progression. J Biol Chem 2011;286:34457-34467.

15 Bi M, Ittner A, Ke YD, Gotz J, Ittner LM: Tautargeted immunization impedes progression of neurofibrillary histopathology in aged P301L tau transgenic mice. PLoS One 2011; 6:e26860.

16 Troquier L, Caillierez M, Burnouf S, Fernandez-Gomez FJ, Grosjean MJ, Zommer N, Sergeant N, Schraen-Maschke S, Blum D, Buee L: Targeting phospho-Ser422 by active tau immunotherapy in the THY-Tau22 mouse model: a suitable therapeutic approach. Curr Alzheimer Res 2012;9:397-405.

17 Kfoury N, Holmes BB, Jiang H, Holtzman DM, Diamond MI: Trans-cellular propagation of tau aggregation by fibrillar species. J Biol Chem 2012;287:19440-19451.
18 d'Abramo C, Acker CM, Jimenez HT, Davies $P$ : Tau passive immunotherapy in mutant P301L mice: antibody affinity versus specificity. PLoS One 2013;8:e62402.

19 Rosenmann H, Grigoriadis N, Karussis D, Boimel M, Touloumi O, Ovadia H, Abramsky O: Tauopathy-like abnormalities and neurologic deficits in mice immunized with neuronal tau protein. Arch Neurol 2006;63:14591467.

20 Shin J, Kepe V, Barrio JR, Small GW: The merits of FDDNP-PET imaging in Alzheimer's disease. J Alzheimers Dis 2011;26(suppl 3):135-145.

21 Fodero-Tavoletti MT, Okamura N, Furumoto S, Mulligan RS, Connor AR, McLean CA, Cao D, Rigopoulos A, Cartwright GA, O’Keefe G, Gong S, Adlard PA, Barnham KJ, Rowe CC, Masters CL, Kudo Y, Cappai R, Yanai K, Villemagne VL: 18F-THK523: a novel in vivo tau imaging ligand for Alzheimer's disease. Brain 2011;134:1089-1100.

22 Zhang W, Arteaga J, Cashion DK, Chen G, Gangadharmath U, Gomez LF, Kasi D, Lam C, Liang Q, Liu C, Mocharla VP, Mu F, Sinha A, Szardenings AK, Wang E, Walsh JC, Xia C, Yu C, Zhao T, Kolb HC: A highly selective and specific PET tracer for imaging of tau pathologies. J Alzheimers Dis 2012;31:601-612.

23 Chien DT, Bahri S, Szardenings AK, Walsh JC, Mu F, Su MY, Shankle WR, Elizarov A, Kolb HC: Early clinical PET imaging results with the novel PHF-tau radioligand [F-18]T807. J Alzheimers Dis 2013;34:457-468.
24 Zakaib GD: http://www.alzforum.org/new/ detail.asp?id=3386;2013.

25 Lin YJ, Koretsky AP: Manganese ion enhances T1-weighted MRI during brain activation: an approach to direct imaging of brain function. Magn Reson Med 1997;38:378-388.

26 Koretsky AP: Is there a path beyond BOLD? Molecular imaging of brain function. Neuroimage 2012;62:1208-1215.

27 Watanabe T, Natt O, Boretius S, Frahm J, Michaelis T: In vivo 3D MRI staining of mouse brain after subcutaneous application of $\mathrm{MnCl}_{2}$. Magn Reson Med 2002;48:852-859.

28 Wadghiri YZ, Blind JA, Duan XH, Moreno C, Yu X, Joyner AL, Turnbull DH: Manganeseenhanced magnetic resonance imaging (MEMRI) of mouse brain development. NMR Biomed 2004;17:613-619.

29 Bertrand A, Khan U, Hoang DM, Novikov DS, Krishnamurthy P, Rajamohamed Sait HB, Little BW, Sigurdsson EM, Wadghiri YZ: Non-invasive, in vivo monitoring of neuronal transport impairment in a mouse model of tauopathy using MEMRI. Neuroimage 2013; 64:693-702.

30 Little BW, Khan U, Rajamohamed Sait H, Hoang DM, Bertrand A, Wadghiri YZ, Sigurdsson EM: Tau immunotherapy improves axonal transport as detected in vivo by manganese-enhanced magnetic resonance imaging. Alzheimers Dement 2012; 8:38. 